\title{
EL APOYO SOCIAL DE FAMILIA Y AMISTADES COMO FACTORES DETERMINANTES DE LAS PRÁCTICAS DEPORTIVAS JUVENILES
}

SOCIAL SUPPORT OF FAMILY AND FRIENDS AS YOUTH SPORT PRACTICES' DETERMINANTS

María Jesús Monteagudo Sánchez ${ }^{1}$

\section{Resumen}

Las prácticas deportivas juveniles se hallan condicionadas por factores psicosociales, capaces de influir en las conductas deportivas de los jóvenes en direcciones opuestas, como facilitadores, promoviendo la actividad deportiva, o como inhibidores, impidiendo o dificultando dicha práctica. El apoyo social es uno de los factores sociales determinantes de las prácticas deportivas juveniles más destacados en la literatura científica. La familia y las amistades son los agentes responsables de este apoyo social más representativos. El estudio de las prácticas deportivas de una muestra de jóvenes españoles permite constatar el papel esencial que los progenitores y el grupo de iguales desempeñan en la promoción de prácticas deportivas regulares, lo que favorece la instauración de estilos de ocio juveniles físicamente activos y duraderos. Los resultados confirman que los jóvenes más predispuestos a desarrollar patrones de práctica deportiva regular pertenecen a familias que valoran el ámbito deportivo y brindan a sus hijos apoyo emocional en este ámbito; y también a aquellos cuyas amistades practican deporte y les animan a continuar realizando este tipo de actividades.

Palabras claves: ocio deportivo juvenil; factores determinantes; apoyo social; familia; amistades; adherencia deportiva

\section{Abstract}

Youth sports practices are conditioned by psychosocial factors, capable of influencing youth's sports behaviors in opposite directions; as facilitators, sports practices are able to promote sports participation, as inhibitors, preventing or hindering sports activity. Social support is one of the most prominent social determinants of youth sports practices in scientific literature. Family and friends are the furthermost representative agents, responsible for social support. A sample of Spanish young people in the field of the study of sports practices confirms the essential role that parents and peers play in promoting regular sports practices, encouraging the expansion of physically active and durable youth leisure styles. The results confirm that young people are more likely to develop regular sport patterns when they belong to families that value the sports field and provide emotional support to their children's sports behavior, and whose friends also play sports and encourage them to continue to practice these activities.

Keywords: youth sport leisure; sport social determinants; social support; family; friends; sport adherence

Fecha de recepción: 23 de octubre de 2015

Fecha de aprobación: 3 de marzo de 2016

Para citar este artículo:

Monteagudo, M.J. (2016). El apoyo social de familia y amistades como factores determinantes de las prácticas deportivas juveniles. Lúdica Pedagógica, (23), 9-18.

1 Directora de la Cátedra Ocio y Conocimiento del Instituto de Estudios de Ocio de la Universidad de Deusto. Miembro del equipo oficial "Ocio y Desarrollo humano" (Ref. IT 587) de dicho instituto y doctora en Ocio y Desarrollo Humano por dicha universidad. Correo electrónico: mjmonte@deusto.es 


\section{INTRODUCCIÓN}

La importancia de la práctica deportiva como alternativa de ocio juvenil vinculada a la salud así como la creciente tendencia al sedentarismo detectada en las sociedades contemporáneas explican el interés que despierta en las instituciones públicas el conocimiento de los factores que predisponen, facilitan y refuerzan las prácticas deportivas juveniles y su mantenimiento a lo largo de la vida. Cada vez son más los estudios que asocian la óptima consecución de beneficios a una práctica regular y estable en el tiempo (Pieron, 2004). Por ello, se recomienda el cultivo de un estilo deportivo que suponga la incorporación voluntaria y temprana de la práctica deportiva en los hábitos de vida, con el suficiente grado de adherencia como para beneficiarse de las condiciones positivas de la acción deportiva regular y mantenerla en etapas posteriores de la vida (Fernández, Contreras y Fernández-Quevedo 2002).

La historia deportiva (Monteagudo, 2014) de cada persona se configuran a partir de las constantes decisiones que esta adopta sobre sus prácticas; en ocasiones, reafirmando su deseo de continuar una actividad; en otras, abandonando o sustituyendo una práctica por otra. La revisión de la literatura evidencia la existencia de variables personales y sociales denominadas factores determinantes de la práctica deportiva (Salmon, Owen, Crawford, Bauman y Sallis, 2003) por su capacidad para ejercer su influjo sobre las conductas deportivas y su evolución en direcciones opuestas; bien como facilitadores, promoviendo la actividad deportiva; bien como inhibidores o barreras para la práctica deportiva (Nahas, Goldfine y Collins, 2003).

Este artículo centra su atención en uno de los determinantes sociales de las prácticas deportivas juveniles más destacados en la literatura científica: el apoyo social. La investigación subraya la incidencia del apoyo de las personas significativas como uno de los factores procedentes del contexto con más influencia en las decisiones que las y los jóvenes adoptan sobre su participación deportiva; más concretamente, se resalta el papel de la familia y de las amistades. Conocer el impacto de estas figuras de referencia resulta primordial ante la evidencia de que sus roles, sobre todo en el caso de los progenitores, se pueden modelar y modificar para garantizar la incidencia positiva de su influjo sobre la práctica deportiva juvenil.
Los párrafos siguientes ofrecen una revisión de los principales hallazgos científicos habidos en torno al constructo apoyo social como factor determinante de las prácticas deportivas juveniles, sus expresiones e impactos en el caso del apoyo procedente de la familia y del grupo de iguales.

\section{REVISIÓN DE LA LITERATURA}

El concepto apoyo social ha orientado los esfuerzos de numerosos investigadores que han revisado la incidencia positiva de las relaciones y vínculos sociales en la evolución de las prácticas deportivas. Este concepto se entiende como cualquier conducta que ayude a una persona a conseguir los objetivos o resultados que se ha marcado (Rees y Hardy, 2000). Según las teorías que respaldan el influjo del apoyo social, cualquier ayuda procedente de los agentes sociales significativos, es decir, de los denominados "los otros significativos" (familia, amistades, educadores, entrenadores, etc.), está positivamente relacionada con la práctica deportiva. Por esta razón, el número de amistades, la estructura familiar o el número de contactos sociales mantenidos pueden ser indicadores importantes a la hora de determinar el apoyo social del que dispone una persona para hacer deporte (Spainer y Allison, 2001).

El apoyo social emerge como uno de los determinantes fundamentales de la práctica deportiva, tanto por su valor explicativo como por su capacidad para predecir las conductas deportivas (Duncan S. C., Duncan T. E., y Strycker, 2005; Beets, Pitteti y Forlaw, 2007). La existencia de apoyos sociales ejerce una influencia positiva sobre las conductas deportivas, de manera que está positivamente relacionada con la práctica deportiva y las decisiones de continuidad o abandono que se adoptan (Spainer y Allison, 2001; Gabriele, Gill, Harber y Fisher, 2005). La hipótesis que subyace a este planteamiento es que el apoyo social derivado de las personas significativas incrementa la motivación y, a través de esta variable, promueve las conductas deportivas.

Estudios recientes, realizados con población adolescente, corroboran el impacto del apoyo social en las conductas deportivas a través de la motivación (Gabriele et al., 2005). El apoyo social actúa como refuerzo positivo que favorece la práctica deportiva a través de la motivación; preferentemente a través de su relación con el disfrute y con el deseo de continuar comprometido con la acti- 
vidad (Gabriele et al., 2005). Por el contrario, cuando la influencia de los agentes sociales se torna en presión se produce un descenso de la motivación que hace que la práctica se perciba en términos más de obligación que de práctica deseada y satisfactoria.

El estudio del constructo apoyo social y sus consecuencias requiere identificar no solo las fuentes de las que procede el apoyo social, sino también el tipo de apoyo social proporcionado. La bibliografía sobre el tema sugiere la existencia de tres tipos de apoyo esenciales en el ámbito deportivo: emocional, informacional, instrumental (Duncan S. C., et al., 2005). El apoyo emocional se traduce en sentimientos de confianza y cariño mostrados, por ejemplo, mediante la asistencia o presencia de personas significativas durante la actividad deportiva; el apoyo informacional adquiere la forma de información o consejos; tiene lugar cuando el agente, origen del apoyo, inicia una conversación sobre la actividad deportiva, su evolución o muestra el interés explícito acerca de algún aspecto de la actividad. Finalmente, el apoyo instrumental tiene lugar cada vez que un agente social de referencia contribuye a la práctica deportiva mediante aportaciones económicas y/o materiales, bajo la forma de equipamiento deportivo (material, ropa, etc.) o solventando cuestiones de movilidad a través del transporte, entre otras. Los estudios realizados sugieren que el apoyo emocional es el más efectivo para la continuidad de las prácticas deportivas. La presencia de los agentes sociales de referencia durante la realización de las actividades deportivas es uno de los principales refuerzos, cuya presencia correlaciona positivamente con el nivel de práctica y su evolución (Duncan S. C., et al., 2005).

Gráfico 1. Relaciones entre apoyo social y conducta deportiva a través de la motivación

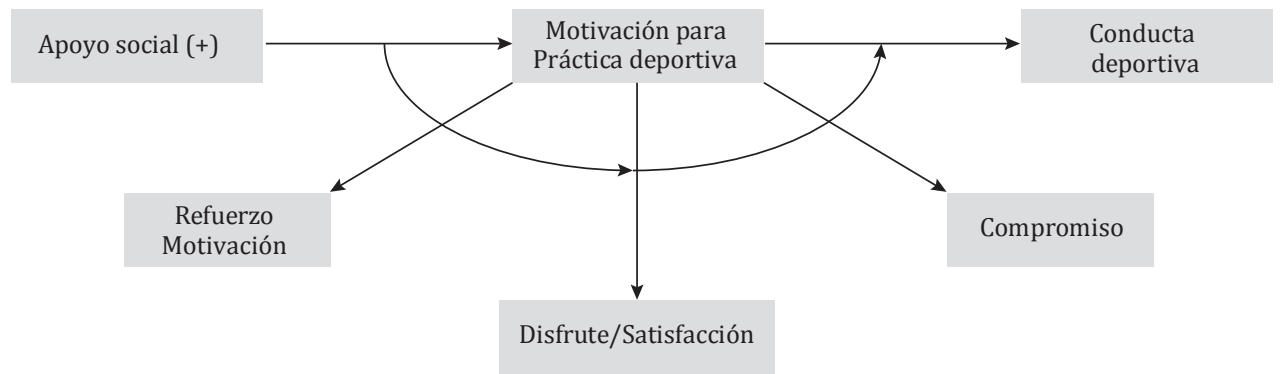

Fuente: Elaborado a partir de Gabriele et al., 2005, p. 213.

Sin embargo, no puede obviarse el carácter dinámico del apoyo social, tanto de las fuentes como de los tipos de apoyo social percibidos. Muchos autores sugieren, por ejemplo, la presencia de cambios importantes relacionados con la edad (Sallis, Taylor, Dowda, Freedson y Pate, 2002). El apoyo percibido se reduce a medida que aumenta la edad; los apoyos de tipo emocional son más comunes en edades tempranas, durante la infancia y adolescencia, mientras que refuerzos de tipo informativo (hablar sobre el tema, proporcionar consejos, sugerencias, etc.) son formas más habituales en edades más tardías (Armstrong y Welsman, 1997). El género es otra de las variables sociodemográficas que incide en la percepción del apoyo social (Arm- strong y Welsman, 1997). Las mujeres demandan más apoyo en aspectos relacionados con el transporte y, en general, perciben más conductas de apoyo social que los hombres. También el estatus socioeconómico marca diferencias significativas en la percepción de conductas y tipos de apoyo. Los jóvenes deportistas pertenecientes a familias con nivel socioeconómico alto percibían más ayudas de todo tipo (asistencia, hacer deporte con agentes significativos, transporte) que los pertenecientes a familias menos acomodadas. Estos últimos acusaban, sobre todo, menos apoyo emocional, en términos de asistencia a actividades deportivas por parte de sus agentes de referencia (Armstrong y Welsman, 1997). 
La incidencia de la familia y las amistades en las prácticas deportivas juveniles

La familia constituye el primer marco de referencia en la vida de las personas. El ámbito del deporte no es una excepción. Para algunos autores, la formación de actitudes y preferencias hacia el deporte así como la consolidación del atractivo y el compromiso con la participación en este ámbito depende, en buena medida, del entorno familiar (Castillo y Sáenz-López, 2007; Fernández, 2007). Las y los hijos que perciben el apoyo de sus padres muestran mayores niveles de disfrute, implicación, inversión personal y compromiso con la práctica de su deporte (Rusbult, 1980). El apoyo de la familia a la práctica deportiva de sus hijos se plantea como un factor clave para el inicio y continuidad de las conductas deportivas (Fernández, 2007). Estudios recientes confirman que los hijos de padres que conceden bastante o mucha importancia a este ámbito están más predispuestos a desarrollar conductas no sedentarias (Sanz, Ponce de León y Valdemoros, 2012). En esta misma línea, Hohepa, Scragg, Schofield, Kolt y Schaaf (2007) confirman que un apoyo familiar escaso al ámbito deportivo reduce de forma considerable las posibilidades de que el hijo/a sea físicamente activo tras el horario lectivo. Sin embargo, no hay conclusiones claras acerca del impacto de los hábitos deportivos de los progenitores en las prácticas deportivas de los hijos, la importancia de hacer deporte en familia o el rol que pueden desempeñar el padre y la madre a este respecto. Algunos estudios sugieren el impacto diferencial de estas figuras en la práctica deportiva de los hijos. Sukys, Majauskienè, Cesnaitiene y Karanauskiene (2014) afirman que mientras las hijas se ven positivamente influenciadas por ambos progenitores, los hijos se muestran especialmente influidos por la figura paterna. Abarca-Sos, Zaragoza, Martín-Albo, y García-González (2014) defienden el papel central de la familia en el fomento de la práctica deportiva de los hijos, pero principalmente del padre, y destacan su impacto en la percepción de competencia y en los sentimientos de disfrute. Subrayan que la incidencia positiva de la figura paterna se produce al margen del apoyo social de la madre.

Respecto al grupo de iguales es importante destacar que el fuerte componente social del deporte adquiere, si cabe, un talante aún más marcado durante la juventud. Algunos estudios demuestran que los jóvenes con estilos de ocio físicamente activos tienen también amigos que practican deporte de forma habitual (Andersen y Wold, 1992), lo cual confirma así la incidencia de la práctica deportiva de las amistades en la participación deportiva juvenil. Según Pieron y Ruiz Juan (2010), el grupo de iguales puede ser clave en las decisiones que las y los jóvenes toman sobre el inicio, continuidad, sustitución o abandono de sus prácticas deportivas, decisiones que dependen de lo que sus amistades hagan a este respecto.

El ámbito deportivo es el contexto ideal para hacer amistades y mantenerlas. "Hacer amigos" es uno de los principales motivos que impulsan la participación deportiva entre los jóvenes (Andersen y Wold, 1992). Tampoco hay que olvidar que, en este periodo, los lazos afectivos más fuertes se establecen con el grupo de iguales, cuya aceptación es uno de los aspectos más valorados. Desde este punto de vista, la práctica deportiva es fuente de refuerzo social. Existen estudios que demuestran que una de las principales formas de aceptación social es contar con habilidades en actividades valoradas por el grupo de iguales. Las prácticas deportivas suelen formar parte de estas actividades más apreciadas durante la adolescencia y juventud. De esta forma, "ser bueno en deporte" puede considerarse un factor facilitador de la integración social (Roberts, 1995). Igualmente, el grupo de iguales es fuente de autoestima, en la medida en que los compañeros son el referente para evaluar la calidad de sus actuaciones deportivas y el cauce a través del que recibe alguna retroalimentación sobre su yo físico (Roberts, 1995).

En esta línea, encontramos estudios que confirman que la participación deportiva de las amistades más significativas afectan a las decisiones de práctica deportiva de jóvenes adolescentes urbanos, entre los 13 y 15 años (Raudsepp y Viira, 2000). Weiss y William (2004) señalan que apenas se ha reparado en la incidencia que las amistades pueden tener en la participación deportiva y su evolución en el tiempo. Sin embargo, son un referente incuestionable en la vida deportiva de los jóvenes y los sujetos con quienes comparten gran parte de sus experiencias deportivas. Ntoumanis, Vazou y Duda (2007) encontraron que las amistades pueden ayudar a predecir el compromiso con la práctica deportiva. Una opinión que coincide con los resultados obtenidos por Weiss y Smith (2002), en relación con el papel de la amistad como predictor del compromiso y la continuidad de la práctica deportiva en jugadores de tenis. 
Ambos agentes, familia y amistades ocupan un lugar privilegiado en la literatura especializada como factores determinantes de las conductas deportivas juveniles. El contraste de la fotografía que arroja esta revisión bibliográfica con los resultados obtenidos en la investigación en la que se apoya este artículo, sobre el rol de la familia y las amistades en la evolución de las prácticas deportivas de las y los jóvenes de Bizkaia será clave para esclarecer la importancia de estos agentes en cuanto fuentes principales de apoyo social.

\section{METOdOLOGÍA}

Los resultados presentados en este artículo están asociados a un estudio más amplio titulado Los itinerarios de ocio deportivo. Estudio de los jóvenes de Bizkaia, ${ }^{2}$ centrado en el caso de Bizkaia, uno de los tres territorios históricos que conforman el País Vasco, en el norte de España, el objetivo de esta investigación es identificar la existencia de diferentes patrones evolutivos del ocio deportivo de las y los jóvenes vizcaínos y esclarecer los factores psicosociales determinantes de sus prácticas deportivas, mediante el estudio de sus trayectorias deportivas entre los 10 y los 20 años de edad. El estudio se apoya en un diseño metodológico mixto que conjuga por un lado, información obtenida de fuentes primarias y secundarias; y por otro, técnicas cualitativas como el análisis documental con otras cuantitativas, como es la técnica de la encuesta.

\section{Participantes}

El universo objeto de estudio lo conforma el conjunto de jóvenes de Bizkaia, de ambos géneros, residentes en cualquier municipio de este territorio histórico, con 20 años de edad en el momento en que se lleva a cabo la investigación. La muestra la componen 383 jóvenes que, cumpliendo los criterios previamente indicados, fueron seleccionados entre la población total mediante un muestreo bietápico estratificado por municipios y cuotas de género. El tamaño muestral permite trabajar con un nivel de confianza de $95 \%$ y un error muestral de $+/-5 \%$. La muestra está compuesta por $51,2 \%$ hombres y $48,8 \%$ mujeres, la mayoría (87,7 \%), estudiantes con situaciones académicas y perfiles forma-

2 Tesis doctoral desarrollada por la autora y defendida en diciembre de 2011. tivos diversos. En consecuencia, el estudio empírico de esta investigación se lleva a cabo atendiendo a los siguientes criterios estadísticos:

Tabla 1. Ficha técnica de la investigación

\begin{tabular}{|l|l|}
\hline Universo & $\begin{array}{l}\text { Jóvenes de ambos géneros residentes } \\
\text { en Bizkaia con } 20 \text { años en el momento } \\
\text { del estudio. }\end{array}$ \\
\hline Ámbito geográfico & Territorio Histórico de Bizkaia \\
\hline Tamaño muestral & 383 encuestas \\
\hline Error muestral & $+/-5 \%$ \\
\hline Nivel de confianza & $95 \% \mathrm{Z}=1,96 \mathrm{p}=\mathrm{q}=0,5$ \\
\hline Diseño muestral & $\begin{array}{l}\text { Muestreo bietápico estratificado por } \\
\text { municipios y cuotas de género. }\end{array}$ \\
\hline
\end{tabular}

Fuente: Elaboración propia.

La segmentación del colectivo atendiendo al tipo de relación mantenida con la práctica deportiva (regular, esporádica o ausente) permite el análisis de sus trayectorias deportivas, la identificación de sus itinerarios deportivos y los factores determinantes del inicio y continuidad de su actividad deportiva. Se entiende por práctica deportiva regular, aquella que tiene lugar con una frecuencia igual o superior a dos días por semana y con una duración igual o superior a dos años consecutivos; esporádica, la que no alcanza los parámetros considerados para la práctica regular; y ausente, aquella que caracteriza a los jóvenes que hacen de la ausencia de práctica deportiva uno de los rasgos distintivos de sus estilos de ocio.

Tabla 2. Aproximación a la práctica deportiva entre 1995-2005

\begin{tabular}{|l|c|c|}
\hline & N & $\%$ \\
\hline Regulares & 221 & $57,7 \%$ \\
\hline Esporádicos & 82 & $21,4 \%$ \\
\hline No practicantes & 80 & $20,9 \%$ \\
\hline Total & 383 & $100,0 \%$ \\
\hline
\end{tabular}

Fuente: Elaboración propia.

\section{Instrumento}

Esta investigación se apoya en un cuestionario elaborado ad hoc para el estudio. El diseño y contenidos del cuestionario buscan aportar la información necesaria para describir y comprender la presencia y relación que los jóvenes de la muestra han mantenido con la 
práctica deportiva entre los 10 y los 20 años de edad, desentrañando la posible existencia de patrones que defina sus trayectorias deportivas en términos de itinerario, los factores psicosociales determinantes de su participación deportiva y de sus decisiones de inicio, continuidad o abandono. La herramienta denominada Cuestionario para la identificación de los itinerarios deportivos juveniles, factores promotores de la práctica deportiva regular y su continuidad, incluye seis apartados dedicados a variables sociodemográficas o de identificación: resumen retrospectivo de la práctica deportiva entre los 10 a los 20 años; práctica deportiva regular; práctica deportiva esporádica; ausencia de práctica deportiva y valoración personal del entorno social en el que se exploran las opiniones de los jóvenes en relación con el impacto del entorno familiar, relacional, educativo y comunitario en sus trayectorias deportivas. Este artículo se apoya en los resultados obtenidos en este último apartado, valoración del entorno, y centra su interés en la familia y el grupo de iguales al emerger en la literatura científica, como los dos agentes con mayor influjo en las prácticas deportivas juveniles.

\section{Procedimiento}

Elaborada la herramienta, se lleva a cabo un pre-test de 25 encuestas, con resultados positivos que permiten iniciar la administración del cuestionario definitivo de carácter anónimo. El trabajo de campo se inicia en mayo de 2008 y se extiende durante los tres meses siguientes hasta finales de julio de este mismo año. La administración de los 383 cuestionarios es realizada por un equipo de encuestadores, previamente formados mediante sesiones específicas. Tras el trabajo de campo y el proceso de depuración de los cuestionarios se procede a la explotación de los datos con el programa sPSS (versión 19.0). La aplicación de estadísticos descriptivos y de correlación permite conjugar la información de conjunto, obtenida del total de los jóvenes de la muestra con información sectorial específica de cada grupo de jóvenes planteados en función de su relación con la práctica deportiva: regular, esporádica o ausente. De esta forma ha sido viable la identificación de los itinerarios de ocio deportivo de este colectivo y los factores promotores implicados en el inicio y continuidad de sus prácticas deportivas.

\section{ANÁLISIS DE RESULTADOS}

El cuestionario empleado en esta investigación dedica todo un apartado a analizar la percepción que las y los jóvenes de la muestra tienen sobre la incidencia del entorno familiar y relacional en sus decisiones sobre la práctica deportiva. Con esta información se pretende esclarecer el papel de la familia y el grupo de iguales en las prácticas deportivas juveniles, evaluando el influjo de estos agentes en las decisiones que las y los jóvenes toman respecto al inicio, continuidad o abandono de sus prácticas deportivas. Los siguientes párrafos analizan los resultados obtenidos en relación con los cuatro contextos considerados.

\section{La familia}

Aunque los agentes familiares con capacidad para incidir en el desarrollo deportivo no son exclusivamente los progenitores, en este artículo, la atención se centrará en ellos por constituir una pieza clave en los procesos de socialización con el deporte. El objetivo es determinar su papel como factor determinante de las prácticas deportivas juveniles. Se indaga la existencia de potenciales diferencias entre las familias de los jóvenes practicantes regulares, esporádicos y no practicantes en relación con la importancia, valores y otros aspectos relacionados con la práctica deportiva. De existir, tales diferencias podrían explicarse las diferentes formas de aproximación a la actividad deportiva (regular, esporádica o ausente) elegida por cada joven. Los siguientes apartados muestran un extracto de los principales resultados obtenidos acerca de estas cuestiones.

Importancia y valores atribuidos a la práctica deportiva en la vida familiar

A través de las opiniones de las y los jóvenes implicados, conocemos la importancia que sus familias atribuyen a la práctica deportiva, los principales valores que le atribuyen así como el contacto que la familia mantiene con este ámbito de manera individual y como espacio de encuentro con su hijo/a. Los datos revelan que existe una relación estadísticamente significativa entre la importancia que otorga la familia a la actividad deportiva y la participación en este ámbito. La mayoría de los jóvenes $(55,7 \%)$ opina que sus familias conceden importancia a la práctica deportiva. Pero esta afirmación es más obvia entre las y los jóvenes con práctica deportiva regular; siete de cada diez jóvenes de este colectivo pertenece a familias que otorgan importancia 
al deporte. Por el contrario, la mayoría de los jóvenes no practicantes $(71,3 \%)$ opina que sus familias no conceden importancia a este ámbito.

En cuanto a los valores atribuidos a la práctica deportiva por parte de las familias observamos que "pasarlo bien" es el aspecto más importante para las familias con practicantes deportivos regulares, mientras que las familias de jóvenes no practicantes no destacan ningún valor asociado a la actividad deportiva. La adquisición de habilidades y el triunfo son aspectos más apreciados por las familias de los jóvenes con prácticas deportivas esporádicas.

Prácticas deportivas de y con la familia

A pesar de que la valoración de la práctica deportiva es muy positiva por parte de las familias de la mayoría de las y los jóvenes de la muestra, los resultados sobre el nivel de práctica de dichas familias revelan que la mayor parte $(60,6 \%)$ no realiza ningún tipo de práctica. Aun así, la ausencia de práctica se muestra especialmente notoria en las familias de jóvenes no practicantes, mientras que la práctica deportiva es más frecuente entre los miembros de las familias cuyos hijos practican deporte, de manera esporádica y regular. Aún más escaso es el porcentaje de jóvenes cuyas familias hacen deporte con sus hijos. Concretamente, el 7,6 \% de los jóvenes afirma que sus familias han compartido con ellos la actividad deportiva. Este porcentaje alcanza el 11,3\% entre los jóvenes con práctica deportiva regular. Aunque estadísticamente la relación de ambas variables no resulta significativa, los resultados indican que, para la mayoría de estos jóvenes que han practicado deporte en familia $(58,6 \%)$, la vivencia de esta experiencia conjunta le ha animado a practicar deporte por su cuenta. Este influjo positivo resulta más evidente entre los jóvenes con práctica regular (68\%).

Percepción de apoyo familiar y tipos de apoyo a la práctica deportiva juvenil

El apoyo de la familia a la práctica deportiva de los hijos que resultaba un factor clave en la literatura también los es para las y los jóvenes de este estudio. El 67,1\% afirma percibirlo. Ser acompañados por sus padres a los entrenamientos y que estos les animaran a seguir practicando ha sido importante, en opinión de estos jóvenes, para el desarrollo de su vida deportiva. Los resultados confirman que la relación apoyo familiarpráctica deportiva es estadísticamente significativa.
La percepción del apoyo familiar es más notoria entre quienes practican deporte regularmente $(86,4 \%)$. Entre practicantes esporádicos, este porcentaje desciende hasta un $62,2 \%$, mientras que entre jóvenes no practicantes prevalece la ausencia de percepción de apoyo familiar para su actividad deportiva (37,5 \%). También es significativo que la mayoría de los jóvenes que no ha incluido la práctica deportiva en sus repertorios de ocio no sepa o no conteste a esta cuestión del apoyo familiar $(43,8 \%)$. Se confirma, por tanto, una relación directa entre percepción de apoyo familiar y tipo de aproximación a la práctica deportiva; de manera que cuanto mayor es la implicación (regular) con la práctica deportiva, mayor es el apoyo familiar percibido; mientras que cuanto menor es el contacto con la actividad deportiva (ausente o esporádico), menor es el apoyo que se percibe procedente de la familia.

Constatado el papel de la familia como fuente de apoyo social de primer orden en materia deportiva, se busca ahora determinar el tipo de apoyo que los padres proporcionan; intentando desentrañar si la naturaleza del apoyo que reciben los practicantes regulares es diferente del que reciben los jóvenes con práctica esporádica. La hipótesis que se defiende es que los practicantes regulares gozan, en mayor medida que los practicantes esporádicos, del apoyo emocional de sus familias. Los resultados corroboran esta hipótesis e indican que la relación entre el tipo de apoyo familiar y la práctica deportiva es estadísticamente significativa. El apoyo familiar más habitual es emocional (36,6\%) y se percibe entre los jóvenes a través de la presencia de la familia en entrenamientos, partidos, torneos u otras competiciones organizadas. Este tipo de apoyo es percibido especialmente por jóvenes con práctica regular (47,1\%). Los jóvenes con práctica esporádica perciben, en mayor medida que el resto, un apoyo informacional $(43,1 \%)$; es decir, perciben el interés de los familiares por la actividad deportiva, sus resultados o evolución. Este apoyo se materializa también a través de consejos y opiniones sobre la actividad, técnicas o dificultades que plantea. El apoyo instrumental (aportación de medios materiales, económicos u organizativos) apenas resulta relevante para los jóvenes de la muestra (5,8\%).

\section{Amistades}

El objetivo de este apartado es explorar la influencia de las amistades en el desarrollo deportivo juvenil. Se pretende comprobar si rodearse de amistades que valoran 
la práctica deportiva y la llevan a cabo con asiduidad puede marcar una diferencia relevante en cuanto a la interacción mantenida con el ámbito deportivo y la trayectoria deportiva realizada.

\section{El valor del deporte entre las amistades}

Prácticamente la mitad de las y los jóvenes de la muestra considera que la actividad deportiva es importante para sus amistades $(49,1 \%)$. Los resultados indican que existe una relación estadísticamente significativa entre la importancia que las amistades atribuyen a la práctica deportiva y el tipo de participación deportiva de las y los jóvenes del estudio. El 61,5\% de los practicantes regulares afirma que sus amistades otorgan importancia al deporte; mientras que siete de cada diez jóvenes pertenecientes al grupo de jóvenes que no practican deporte consideran que el deporte no es relevante para sus amistades. Se confirma así la existencia de posturas similares o idénticas entre las amistades en lo que concierne al ámbito deportivo (valores y conductas deportivas). Son las amistades de los jóvenes practicantes más vinculados a la actividad deportiva (regulares) quienes más la valoran; y al revés, las amistades de quienes no practican son quienes menos importancia otorgan a la acción deportiva.

\section{Prácticas deportivas con las amistades}

Se trata ahora de descubrir si los jóvenes cuyas amistades otorgan importancia a la práctica deportiva, practicantes regulares, son también quienes llevan a cabo sus actividades deportivas con sus amistades. Efectivamente, son las y los practicantes regulares quienes comparten, en mayor medida, sus prácticas con las amistades $(62,4 \%)$. Prácticamente, el mismo porcentaje que afirma que para sus amistades el deporte es importante $(49,1 \%)$ indica ahora que realiza sus actividades deportivas con estas amistades $(47,8 \%)$. La relación que se establece entre la práctica deportiva realizada con las amistades y el tipo de aproximación a la actividad deportiva es estadísticamente significativa.

\section{Opinión sobre influencia de las amistades}

en la práctica deportiva juvenil

Más de la mitad de los jóvenes (52,7 \%) afirma que sus amistades sí han ejercido alguna influencia en su vida deportiva. Partiendo de una relación estadísticamente significativa ( $\operatorname{sig}=0,00$ ) entre percepción del impacto de las amistades y el tipo de acercamiento a la práctica deportiva (regular, esporádico o ausente), se confirma que son los practicantes, especialmente los regulares $(62,4 \%)$, quienes consideran que sus amistades han influido en su práctica deportiva. Por el contrario, siete de cada diez jóvenes no practicantes afirman no haber estado influenciados por sus amistades a la hora de decidir su alejamiento de la práctica deportiva. Aún más interesante es analizar el tipo de impacto que dichas amistades han ejercido en la implicación deportiva juvenil. Los datos corroboran de nuevo la existencia de una relación estadísticamente significativa entre el tipo de relación mantenida con la práctica deportiva (regular, esporádica o ausente) y la naturaleza de la influencia percibida por parte de las amistades. La influencia de las amistades ha sido para algunos jóvenes un aliciente para su actividad deportiva, mientras que para otros, ha favorecido conductas sedentarias, lo que induce su alejamiento de la práctica deportiva. Los practicantes regulares son quienes se muestran más de acuerdo con la idea de que la actividad deportiva de las amistades les animaba a practicar (79\%). Por el contrario, los jóvenes no practicantes opinan que las amistades han incidido en ellos negativamente al no realizar ningún tipo de deporte $(72,2 \%)$.

\section{REFLEXIONES FINALES}

Aunque existe gran controversia en relación con los determinantes sociales de la práctica deportiva, los resultados de este estudio confirman el rol definitivo de la familia (progenitores) y el grupo de iguales como factores determinantes de las prácticas deportivas juveniles por el potencial apoyo que pueden proporcionar al inicio y continuidad de estas conductas, en cuanto agentes sociales significativos.

\section{Sobre la familia}

La distinción entre tres formas distintas de aproximación a la práctica deportiva juvenil (regular, esporádica y ausente) ha resultado especialmente interesante en este estudio porque ha evidenciado el papel fundamental que desempeña la familia en la promoción de la práctica deportiva regular. Una forma de hacer deporte especialmente recomendada por varios motivos. En primer lugar, porque la frecuencia y continuidad que conlleva garantiza la optimización de los múltiples beneficios que se pueden obtener de la práctica deportiva. En segundo lugar, porque esta forma de hacer 
deporte habilita a su protagonista para una experiencia deportiva radicalmente distinta y más intensa que la esporádica. Una experiencia caracterizada por una elevada implicación, compromiso y apego emocional que es directamente proporcional a la satisfacción que propicia su práctica. Es importante subrayar que las prácticas deportivas regulares de las y los jóvenes estudiados mostraban cierta similitud con las manifestaciones de ocio serio descritas por Stebbins (2007).

Esta consideración de la familia como ente promotor de la práctica deportiva regular ha quedado comprobada en este estudio a través de la importancia que los progenitores otorgan a la actividad deportiva, los valores que en ella destacan así como la percepción y naturaleza del apoyo que proporcionan a sus hijos en materia deportiva. Se constata, en primer lugar, que la importancia otorgada a la práctica deportiva por las familias es mayor, cuanto mayor es la implicación deportiva de los jóvenes; esto es, en el caso de la práctica deportiva regular. En segundo lugar, la percepción de apoyo familiar por parte de los jóvenes es también un aspecto que difiere según el tipo de interacción mantenida con la práctica deportiva. Dicha percepción de apoyo es mayor, cuanto mayor es la dedicación a la práctica deportiva (práctica regular). De ahí que los jóvenes practicantes regulares perciban más el apoyo de sus familias que el resto de los grupos (practicantes esporádicos y no practicantes). Además, prevalece entre los jóvenes practicantes regulares la percepción de un apoyo familiar emocional que, tal y como se comprueba en la literatura, resulta el más efectivo, en términos de inicio y continuidad de la participación deportiva (Duncan S. C., et al., 2005). Por contraste, el impacto de la práctica deportiva de la familia y de la existencia de prácticas deportivas compartidas en familia no resulta tan claro en el desarrollo deportivo juvenil.

Sobre las amistades

Las amistades encierran un enorme potencial en la configuración de las historias deportivas, al ser capaces de promover o inhibir la participación deportiva de sus iguales. El componente social de la acción deportiva es fundamental para entender este influjo. Los jóvenes deportistas tienden a rodearse de amigos que comparten con ellos valores similares en relación con la práctica deportiva y con los que poder disfrutar de experiencias en este ámbito. La incidencia de las amistades en la práctica deportiva se hace más obvia cuanto mayor es la dedicación a esta actividad. Para quienes hacen deporte regularmente, las amistades son un factor promotor de la participación deportiva pues la implicación de sus iguales en este ámbito incita a los jóvenes regulares a seguir practicando; en cambio, las amistades que no hacen deporte inhiben la participación deportiva de los jóvenes esporádicos y, especialmente, de los sedentarios. Se puede afirmar, por tanto, que la capacidad de las amistades para favorecer o dificultar la participación deportiva descansa en su poder de retroalimentar las conductas mayoritarias.

En definitiva, se puede afirmar que las y los jóvenes más predispuestos a desarrollar patrones de práctica deportiva regular son aquellos que están:

- arropados por un entorno familiar que valora positivamente el ámbito deportivo y proporciona apoyo emocional a las conductas deportivas de sus hijos,

- insertos en un círculo de amistades, también practicantes deportivos, que les animan a desarrollar este tipo de actividades.

Son muchos los interrogantes que quedan por resolver en torno al papel de la familia y las amistades como principales agentes promotores de la práctica deportiva juvenil a través de las diferentes fórmulas de apoyo social que despliegan. Sin embargo, la confirmación de su influjo en la evolución de las conductas deportivas juveniles invita a reflexionar sobre la necesidad de formar a estos agentes, principalmente a los progenitores, para garantizar que su intervención abogue por la consolidación de estilos de ocio deportivos juveniles satisfactorios y duraderos.

\section{REFERENCIAS}

Abarca-Sos, A., Zaragoza, J., Martín-Albo, J. y GarcíaGonzález, L. (2014). Determinantes para la práctica de actividad física en adolescentes: factores personales, sociales y ambientales que influencian los niveles de actividad física. En Generelo, E., Zaragoza, J., y Julián, J. A. (coord.). Promoción de la actividad física en la infancia y la adolescencia (pp. 69-83). Madrid: CSD.

Andersen, N., y Wold, B. (1992). Parental and peer influences on leisure-time physical activity in young adolescents. Res Q. of Exercise and Sport, 63, 341-348.

Armstrong, N. y Welsman, J. (1997). Young people and physical activity. New York: Oxford University Press. 
Beets, M. W., Pitteti, K. H., Forlaw, L. (2007). The role of self-efficacy and referent specific social support in promoting rural adolescent girls' physical activity. American Journal of Health Behavior, 31(3), 227-237.

Castillo, E., y Sáenz-López, P. (2007). Hábitos relacionados con la práctica de actividad física de las alumnas de la Universidad de Huelva a través de historias de vida. Profesorado. Revista de currículo y formación del profesorado, 11(2), 1-18.

Duncan, S. C., Duncan, T. E. y Strycker, L. A. (2005). Sources and types of social support in youth physical activity. Health Psychology, 24(1), 3-10.

Fernández, E. (2007). Barreras para la práctica deportiva de las mujeres españolas durante la infancia y la juventud. ADOZ. Revista de Estudios de Ocio, 31, 49-57.

Fernández, E., Contreras, O., Sánchez, F., y FernándezQuevedo, C. (2002). Evolución de la práctica de actividad físicay el deporte en las mujeres adolescentes e influencia en la percepción del estado general de salud. Mujeres y actividades físico-deportivas. Revista IcD de Estudios sobre Ciencias del Deporte, 35, 21-60.

Gabriele, J. M., Gill, D. L., Harber, K. D., y Fisher, E. B. (2005). Differentiates roles of social encouragement and social constraint on physical activity behavior, Annals of Behavioral Medicine, 29(3), 210-215.

Hohepa, M., Scragg, R., Schofield, G., Kolt, G. S., y Schaaf, D. (2007). Social support for youth physical activity: Importance of siblings, parents, friends and school support across a segmented school day. International Journal of Behavioral nutrition and Physical activity, 4, 54 doi:10.1186/1479-5868-4-54

Monteagudo Sánchez, M. J. (2014). Factores determinantes de la adherencia deportiva juvenil: aportaciones desde los itinerarios de ocio deportivo. Revista Subjetividades, 14(1), 65-86.

Nahas, M. V., Goldfine, B., y Collins, M. A. (2003). Determinants of physical activity in adolescents and young adults: the basis for high school and college physical education to promote activite lifestyles. Physical Educator, 60(1), 42-56.

Ntoumanis, N., Vazou, S., y Duda, J. L. (2007). Peer-created motivational climate. En S. Jowett, D. Lavallee (eds.), Social Psychology in Sport (pp. 145-156). Champaign: Human Kinetics.

Pieron, M. (2004). Estilo de vida, práctica de actividades físicas y deportivas, calidad de vida. Fitness \& Performance Journal, 3(1), 10-17.

Pieron, M., y Ruiz Juan, F. (2010). Actividad físicodeportiva y salud. Análisis de los determinantes de la práctica en el alumnado de Enseñanza Secundaria. Madrid: Ministério de Educación. Consejo Superior de Deportes.
Pino, J., Dueso, A., y Centro de Investigaciones Sociológicas (cIs) (2001). Prácticas de ocio, cambio cultural y nuevas tecnologías en la juventud española de fin de siglo. Madrid: CIS.

Raudsepp, L., y Viira, R. (2000). Influence of parents' and siblings' physical activity on activity levels of adolescents. European Journal of Physical Education, $5,169-178$.

Rees, T., y Hardy, L. (2000). An investigation of the social support experiences of high-level sports performers. The Sport Psychologist, 14, 327-347.

Roberts, G. (1995). Motivación en el deporte y el ejercicio. Bilbao: Desclée de Brouwer.

Rusbult, C. E. (1980). Commitment and satisfaction in romantic association. A test of the investment model. Journal of Experimental Social Psychology, 16, 172186.

Sallis, J. F., Taylor, W. C., Dowda, M., Freedson, P. S., y Pate, R. R. (2002). Correlators of vigorous physical activity for children in grades 1 through 12: comparing parent reported and objectively measured physical activity. Pediatric Exercise Science, 14, 30-44.

Salmon, J., Owen, N., Crawford, D., Bauman, A., y Sallis, J. F. (2003). Physical activity and sedentary behavior: a population-based study of barriers, enjoyment and preference. Health Psychology, 22, 2, 178-188.

Sanz, E., Ponce de León, A., y Valdemoros, M. A. (2012). Parental predictors of physical inactivity in spanish adolescents. Journal of Sports Science and Medicine, 11(1), 95-101.

Stebbins, R. A. (2007). Serious leisure: A perspective for our time. New Brunswick and London: Transaction Publishers.

Spainer, P. A., y Allison, A. R. (2001). General social support and physical activity: an analysis of the Ontario Health Survey. Canadian Journal of Public Health, 92, 210213.

Sukys, S., Majauskienè, D., Cesnaitiene, V. J., y Karanauskiene, D. (2014). Do parents' exercise habits predict 13-18 year-old adolescents' involvement in sport? Journal of Sports Science y Medicine, 13(3), 522.

Weiss, M. R., y Smith, A. L. (2002). Friendship quality in youth sport: relationship to age, gender and motivation variables. Journal of Sport y Exercise Psychology, 7, 215-233.

Weiss, M. R., y William, L. (2004). The why of youth sport involvement: a developmental perspective on motivational processes. En M. R. Weiss (ed.), Developmental sport and exercise psychology: a life span perspective (pp. 223-268). Morgantown: Fitness Information Technology. 